\title{
Pulmonary arteriovenous fistula diagnosed by contrast echocardiography: a case report of a daughter and a mother
}

\section{Gaoyun Pan ( $853701271 @ q q . c o m$ )}

Wenzhou People's Hospital https://orcid.org/0000-0002-7109-4548

\section{Xinxin Dong}

Wenzhou People's Hospital

Jianguo Xu

Wenzhou People's Hospital

\section{Case report}

Keywords: pulmonary arteriovenous fistula, contrast echocardiography, diagnosis

Posted Date: March 4th, 2020

DOI: https://doi.org/10.21203/rs.3.rs-16024/v1

License: (c) (i) This work is licensed under a Creative Commons Attribution 4.0 International License.

Read Full License 


\section{Abstract}

Background Pulmonary arteriovenous fistula is a rare disease with a direct connection between the pulmonary artery and the vein, and in most cases is congenital. In a proportion of patients, it can cause hypoxemia, cyanosis and dyspnea. The golden standard for the diagnosis of PAVF is pulmonary angiography. We experienced two cases of a daughter and a mother with PAVF diagnosed by contrast echocardiography, which is simple and sensitive for the detection of pulmonary arteriovenous fistula.

Case presentation Case 1:A 22-year-old female was admitted to hospital because of "unconsciousness for 3 hours after sudden seizures".CT showed left frontal cerebral arteriovenous malformation with hemorrhage, a nodule of upper lobe of left lung, arteriovenous malformation possible.Intracranial hematoma removal, arteriovenous malformation resection were performed urgently. Postoperatively, the patient presented severe hypoxemia. Contrast echocardiography showed continuous dense bubbles were visualized in the left heart from the third heart cycle following imaging in the right heart, , suggesting pulmonary arteriovenous fistula. Case 2 : The mother of the first patient, 44-year-old female, with no history of dyspnea, cyanosis,and stroke, was medically screened for suspected pulmonary arteriovenous fistula due to her daughter's disease. Contrast echocardiography also indicated pulmonary arteriovenous fistula.

Conclusions Contrast echocardiography is an excellent tool for the detection of pulmonary arteriovenous fistula. Patients with suspected pulmonary arteriovenous fistula should be examined by chest radiography combined with contrast echocardiography as first line screening tests, especially in patients with severe condition.

\section{Background}

Pulmonary arteriovenous fistula $\mathrm{PAVF} \triangle$ is a rare disease with a direct connection between the pulmonary artery and the vein, and in most cases is congenital. In a proportion of patients, it can cause hypoxemia, cyanosis and dyspnea. The golden standard for the diagnosis of PAVF is pulmonary angiography. We experienced two cases of a daughter and a mother with PAVF diagnosed by contrast echocardiography, which is simple and sensitive for the detection of PAVF.

\section{Case Presentation}

Case 1

A 22-year-old female was admitted to hospital because of "unconsciousness for 3 hours after sudden seizures". Physical Examination: coma, T $37.2{ }^{\circ} \mathrm{C}$ BP 137 / 80mmHg, P 125bpm Sp02 90\%, bilateral pupils of equal size with diameter of $3.5 \mathrm{~mm}$, fixed with light reflect test, auscultation of cardiopulmonary negative, pathological signs were not drawn, cluster of papular lesions on the left breast, slightly rounded, ruby-colored, sharply demarcated from surrounding skin. 
She underwent brain surgery 16 years ago with a history of epilepsy. CT of the brain and chest, CTA of the brain: left frontal arteriovenous malformation with hemorrhage, nodule of upper lobe of left lung, arteriovenous malformation possible, ground glass nodule of lower lobe of right lung, and scattered inflammatory foci in two lungs(Figure 1,2). Laboratory tests: white blood cell count: $24.7 * 10^{\wedge} 9$ / L; neutrophil percentage: $95.7 \%$; red blood cell count: $4.57 * 10^{\wedge} 12$ / L; hemoglobin: $153 \mathrm{~g} / \mathrm{L}$; platelet count: $194 * 10^{\wedge} 9$ / L; C-reactive protein: $1.3 \mathrm{mg} / \mathrm{L}$. Intracranial hematoma removal, arteriovenous malformation resection, and bone plate decompression were performed urgently. Postoperatively, symptomatic supportive treatment such as boost-capacity, sedation, anti-epilepsy, and anti-infection were given. However, the patient's condition further deteriorated, with bilateral pupil dilated, dull light reflection, unstable circulation, hypoxemia, oxygen saturation between $35-50 \%$ under pure oxygen, and $\mathrm{PaO} 2$ of $54 \mathrm{mmHg}$. Repeated blood routine: white blood cell count: $9.8^{*} 10^{\wedge} 9$ / L; neutrophil percentage: 84.2\%; red blood cell count: $3.12 * 10^{\wedge} 12$ / L; hemoglobin: $103 \mathrm{~g} / \mathrm{L}$; platelet count: 196 * $10^{\wedge} 9$ / L. C-reactive protein: $133.9 \mathrm{mg} / \mathrm{L}$. Repeated chest CT revealed worsening of both lung infections. Contrast echocardiography was performed with $10 \mathrm{ml}$ agitated saline injected into left cubital vein, while the apical four chamber view was acquired simultaneously. Contrast echocardiography showed continuous dense bubbles were visualized in the left heart from the third heart cycle following imaging in the right heart, suggesting pulmonary arteriovenous fistula(Figure 3 ). There were no obvious abnormalities in conventional echocardiography, no obvious abnormality on the chest radiography, and no obvious abnormality in the electrocardiogram. Because the patient cannot be moved, pulmonary angiography can't be performed to confirm the diagnosis. Despite symptomatic treatment, the patient died of respiratory failure ultimately.

\section{Case 2}

The mother of the first patient, 44-year-old female, with no history of dyspnea, cyanosis,and stroke, was medically screened for suspected PAVF due to her daughter's disease. Her arterial blood gas analysis showed $\mathrm{PaO} 2$ of $38 \mathrm{mmHg}$ and $\mathrm{SaO} 2$ of $73 \%$ under room air. Though conventional echocardiography showed no abnormal finding, contrast echocardiography revealed that continuous dense bubbles were visualized in the left heart from the third heart cycle following imaging in the right heart, which suggested PAVF(Figure 4). Accordingly, CT pulmonary angiography suggested left pulmonary arteriovenous fistula(Figure 5).

\section{Discussion And Conclusions}

PAVF refers to the direct connection of pulmonary arteries and pulmonary veins, forming a fistula or tumor-like lesion and bypassing capillaries. the disease is mostly congenital, with the incidence of 2-3 per 100,000 [1]. The clinical manifestation vary from being totally absent to severe cyanosis, congestive heart failure, and even fulminant respiratory failure[2,3]; $47-80 \%$ of patients with PAVF have hereditary hemorrhagic telangiectasia (HHT), on the contrary, almost 15 to $35 \%$ of patients with HHT have PAVF[46]. Also know as Rendu-Osler-Weber syndrome, HHT is a condition which is transmitted in autosomal dominant pattern, and manifested by capillary dilatation and bleeding on the surface of the skin and 
mucosa and arteriovenous malformations(AVM) of the lung, brain and liver[7-9]. The first present patient, the daughter, most likely had HHT owing to pimples on the breast and cerebral AVM. Multiple complications of the central nervous system are common and may initially appear in PAVF, such as brain abscess, transient ischemic attack (TIA), migraine, and cerebral infarction, with a incidence of $10-19 \%$ [9]. The present first patient had a history of epilepsy, which could not be ruled out as a complication of central nervous system of PAVF.

The diagnostic method for PAVF includes shunt fraction measurement, chest radiography, contrast echocardiograohy, computed tomography, radionuclide perfusion lung scanning and pulmonary angiography. The golden standard for the diagnosis of PAVF is pulmonary angiography, while contrast echocardiography is a newer noninvasive method that appears to be very simple and sensitive for the detection of PAVF[10]. Contrast echocardiography is an excellent tool for evaluation of intracardiac and intrapulmonary right-to-left shunt(RLS), and is able to identify small shunts even when they are not confirm by other methods[11]. Because the air microbubble's diameter is greater than capillary vessel, the microbubbles can't pass through to left heart in patients without RLS. As long as the bubbles are visualized in the left heart, RLS could be verified, even though several bubbles. Barzilai et al[11] performed contrast echocardiography in nineteen patients with HHT (four with suspected PAVF). Fourteen of the patients with HHT had a positive result, of which eleven patients underwent pulmonary angiography and all had validated PAVF; only six of the 11 patient and eight were found to have abnormality with chest radiography and $\mathrm{PaO} 2$ respectively. Contrast echocardiography probably has a sensitivity of nearly $100 \%$ for detecting PAVF, and has been shown to be more sensitive than plain film and CT [12]. This method needs to inject $10 \mathrm{ml}$ of agitated saline (with $1 \mathrm{ml}$ of air) into a peripheral vein while simultaneously scanning the right and left heart with 2D echocardiography. Both solutions contain microbubbles which can yield strong reflection by ultrasound imaging compared with the normal blood flow [13].

We experienced two cases of a daughter and a mother with PAVF diagnosed by contrast echocardiography. The findings of contrast echocardiography of PAVF are as follows: delayed appearance of microbubbles in the left heart, mostly 3 cardiac cycles after right atrial bubbles is visualized; dense continuous microbubbles in the left heart, with less change with Valsalva manoeuvre; when the shunt is of large amount, the left heart contrast agent is still present when the right heart contrast agent subsides; visualization of bubbles in the pulmonary veins is direct evidence of pulmonary arteriovenous fistula. Typical intracardiac RLS (PFO or ASD) are usually seen in the first three cardiac cycles following the visualization of right atrial microbubbles, and intracardiac RLS amount depends on the pressure between the right and left atrium. Intracardiac RLS occurs only when the right atrial pressure surpass the left atrium, which is obvious with Valsalva manoeuvre[14]. Normally there is a tiny amount of intracardiac RLS by contrast echocardiaography, which is easily distinguished from PAVF. Theoretically, it is supposed that the shunt through pulmonary circulation in PAVF occurs later than that in PFO, which takes more time for the contrast agent to pass from the pulmonary artery through PAVF to the pulmonary vein. Occasionally, the shunt in PAVF could occur within three cardiac cycles, and the shunt in PFO can occur later than three cardiac cycles. The shunt in the present patients with PAVF both happened at the third cardiac cycle. Therefore, PFO and PAVF cannot be reliably differentiated depending on the emerging 
time of shunt alone, while the appearance of dense continuous bubbles is more reliable and specific for the diagnosis of PAVF.

The intrapulmonary shunt revealed by contrast echocardiography requires additional assessment of PAVF, which generally should be digital substraction angiography. We recommend that patients with suspected PAVF should be examined by chest radiography combined with contrast echocardiography as first line screening tests, which can be performed bedside when necessary. If positively, advanced invasive examination such as digital substraction angiography should be take into account to validate the diagnosis. Because of the severity condition, the daughter could not undergo pulmonary angiography and further lobectemy or embolotherapy. The severe hypoxemia of the first present patient may be caused by complication of PAVF and cerebral AVM such as PAVF rupture, Sequelae of hemorrhage of cerebal AVM, or deterioration of lung infection. Unfortunately the severe hypoxemia could not be reversed ultimately, which could have been avoided if the PAVF and cerebral AVM was recognized and treated earlier. For the mother, we advised that she take a pulmonary angiography, if necessarily, surgery or embolotherapy should be considered.

\section{Conclusion}

Contrast echocardiography is an excellent tool for the detection of pulmonary arteriovenous fistula. Patients with suspected PAVF should be examined by chest radiography combined with contrast echocardiography as first line screening tests, especially in patients with severe condition.

\section{Declarations}

\section{Ethics approval and consent to participate}

The case report was approved by the ethics committee of Wenzhou People's Hospital. Written informed consent was obtained.

\section{Consent for publication}

Written informed consent of publication was obtained.

\section{Availability of data and materials}

The data and images used and analysed during the case report are available from the corresponding author on reasonable request.

\section{Competing interests}

The authors declare that they have no competing interests

\section{Funding}


The study was funded by Wenzhou Bureau of Science and Technology $₫$ Y20160471】

\section{Authors' contributions}

GP performed the contrast echocardiography and drafted the manuscript. XD performed the conventional echocardiography. JX collected the patients'clinical history.

\section{Acknowledgments}

We are grateful to the radiologists of the radiology department of the Wenzhou People's Hospital for their invaluable assistance in patients'images collecting.

\section{References}

1.Gossage JR, Kanj G. Pulmonary arteriovenous malformations. A state of the art review. Am J Respir Crit Care Med 1998; 158: 643-61.

2.Allen SW, Whitfield JM, Clarke DR, Sujansky E, et al. Pulmonary arteriovenous malformation in the newborn: a familial case. Pediatr. Cardiol 1993; 14:58-61.

3.Taylor G. Pulmonary arteriovenous malformation: an uncommon cause for cyanosis in the newborn. Pediatr. Radiol 1983;13:339-341.

4. Saboo SS, Chamarthy M, Bhalla S, et al. Pulmonary arteriovenous malformations: diagnosis. Cardiovasc Diagn Ther 2018;8:325-337.

5. Holzer RJ, Cua CL. Pulmonary arteriovenous malformations and risk of stroke. Cardiol Clin 2016;34:241-246.

6. Shovlin CL, Condliffe R, Donaldson JW, et al. British Thoracic Society Clinical Statement on pulmonary arteriovenous malformations. Thorax 2017;72:1154-1163.

7. Faughnan ME, Palda VA, Garcia-Tsao G, et al. International guidelines for the diagnosis and management of hereditary haemorrhagic telangiectasia. J Med Genet 2011;48:73-87.

8. Kane I, Pitt Ford A, Lawton K, et al. Ischaemic stroke in a 21-year-old with hereditary haemorrhagic telangiectasia.Pract Neurol 2016;16:381-384.

9.Lin G, Jiang P, Lou M. Thrombolysis in Ischemic Stroke Patients with Isolate Pulmonary Arteriovenous Malformations. J Stroke Cerebrovasc Dis 2019; 28: e68-e70

10.Cottin V, Dupuis-Girod S, Lesca G,et al. Pulmonary vascular manifestations of hereditary hemorrhagic telangiectasia (rendu-osler disease). Respiration 2007;74:361-78. 
11. Barzilai B, Waggoner AD, Spessert C,et al. Two- dimensional contrast echocardiography in the detection and follow-up of congenital pulmonary arteriovenous malformations. Am J Cardiol 1991; 68:1507-1510.

12.Margreiter J, Dessl A, Mair P, et al. Pulmonary arteriovenous fistula detected with transesophageal contrast echocardiography. J Cardiothorac Vasc Anesth 2001;15:755-757.

13. Fraker TD, Harris PJ, Behar VS, et al. Detection and exclusion of interatrial shunts by two-dimensional echocardiography and peripheral venous injection. Circulation 1979;59:379-384.

14.He L, Cheng G, Du Y, et al. A Case Report on Pulmonary Arteriovenous Fistula with Recurrent Cerebral Infarction. The Heart Surgery Forum 2017;20:E98-101.

\section{Figures}
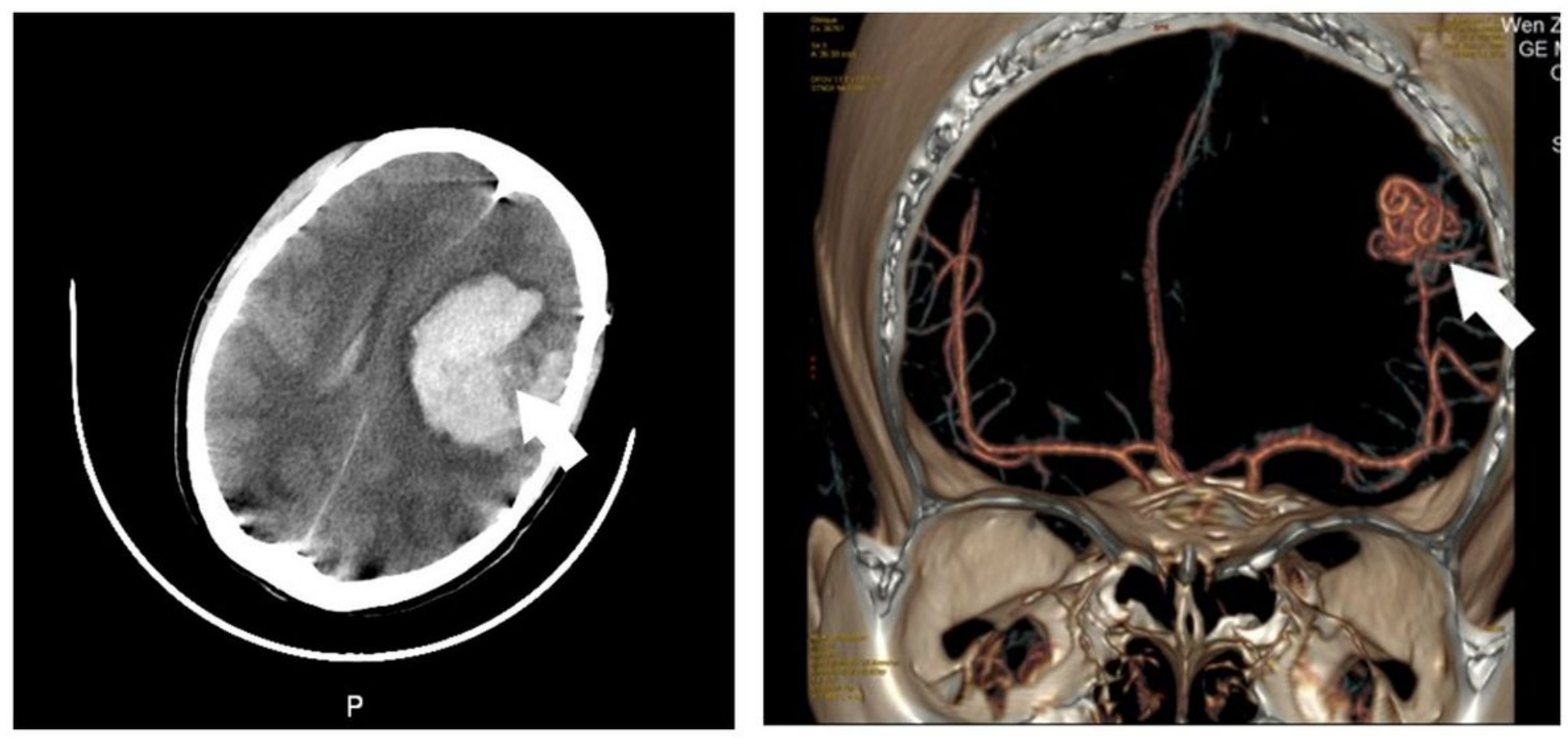

\section{Figure 1}

Left panel: cerebral CT showed left frontal high density shadow indicating hemorrhage(white arrow) ; right panel: 3D reconstruction of cerebral arteries indicating rteriovenous malformation(white arrow). 

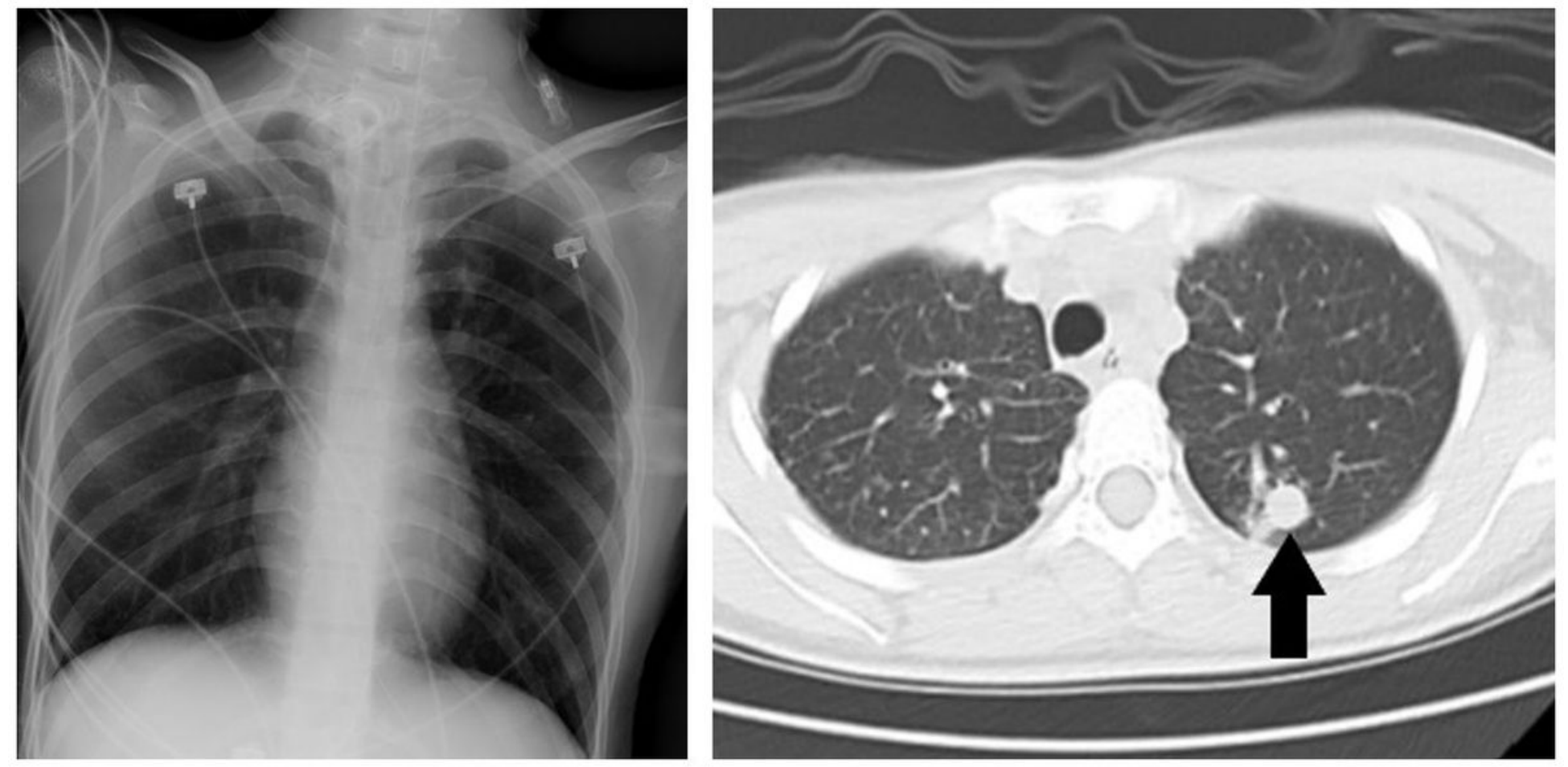

Figure 3

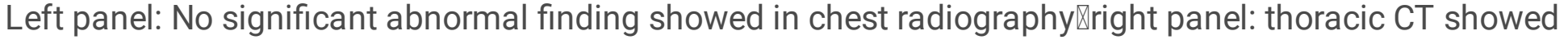
a nodule of the upper lobe of left lung, arteriovenous fistula possible (black arrow). 


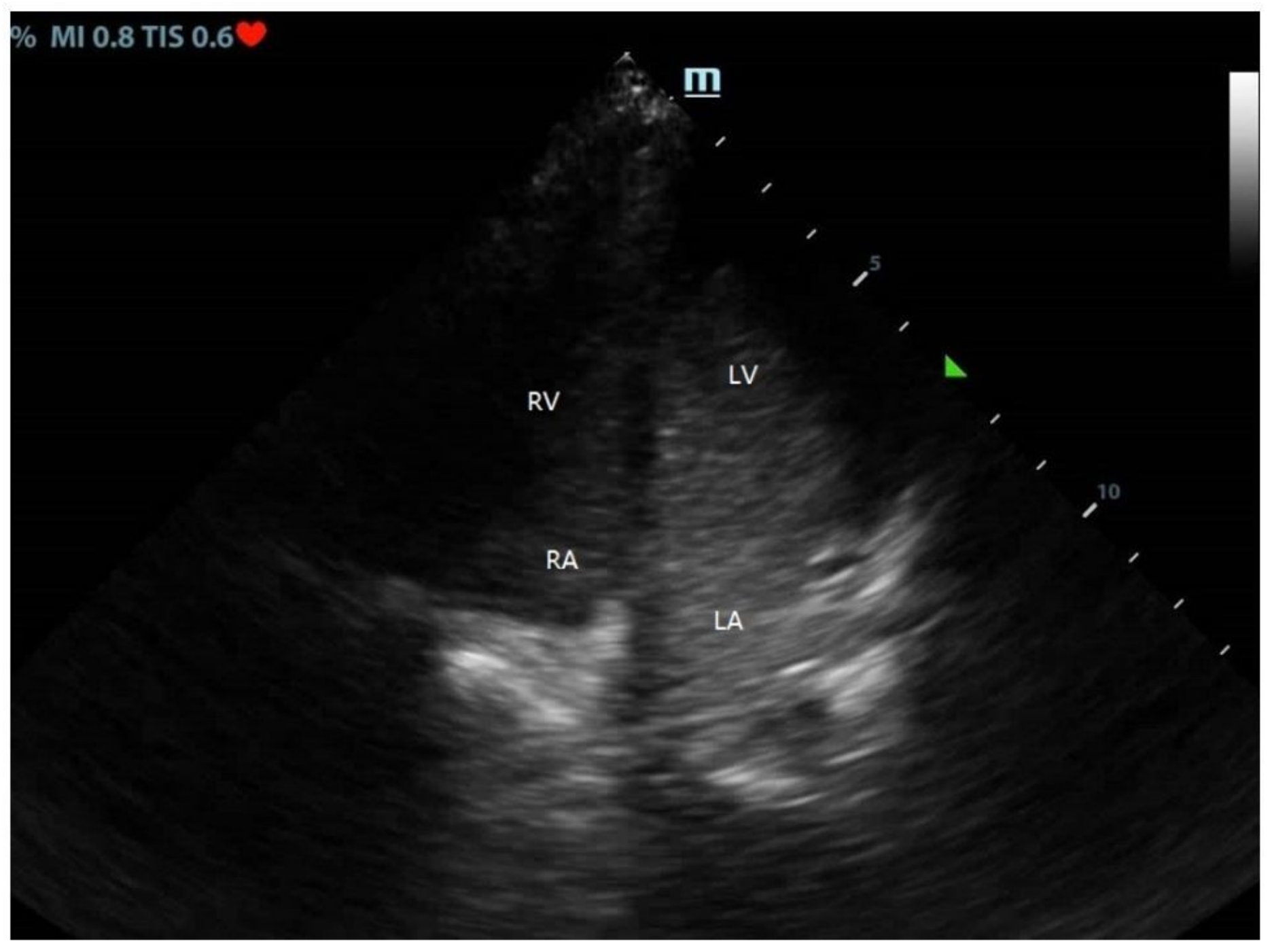

Figure 5

Contrast echocardiography showed dense microbubbles the left heart from the third cardiac cycle following the imaging of microbubbles of the right heart, suggesting pulmonary arteriovenous fistula. 


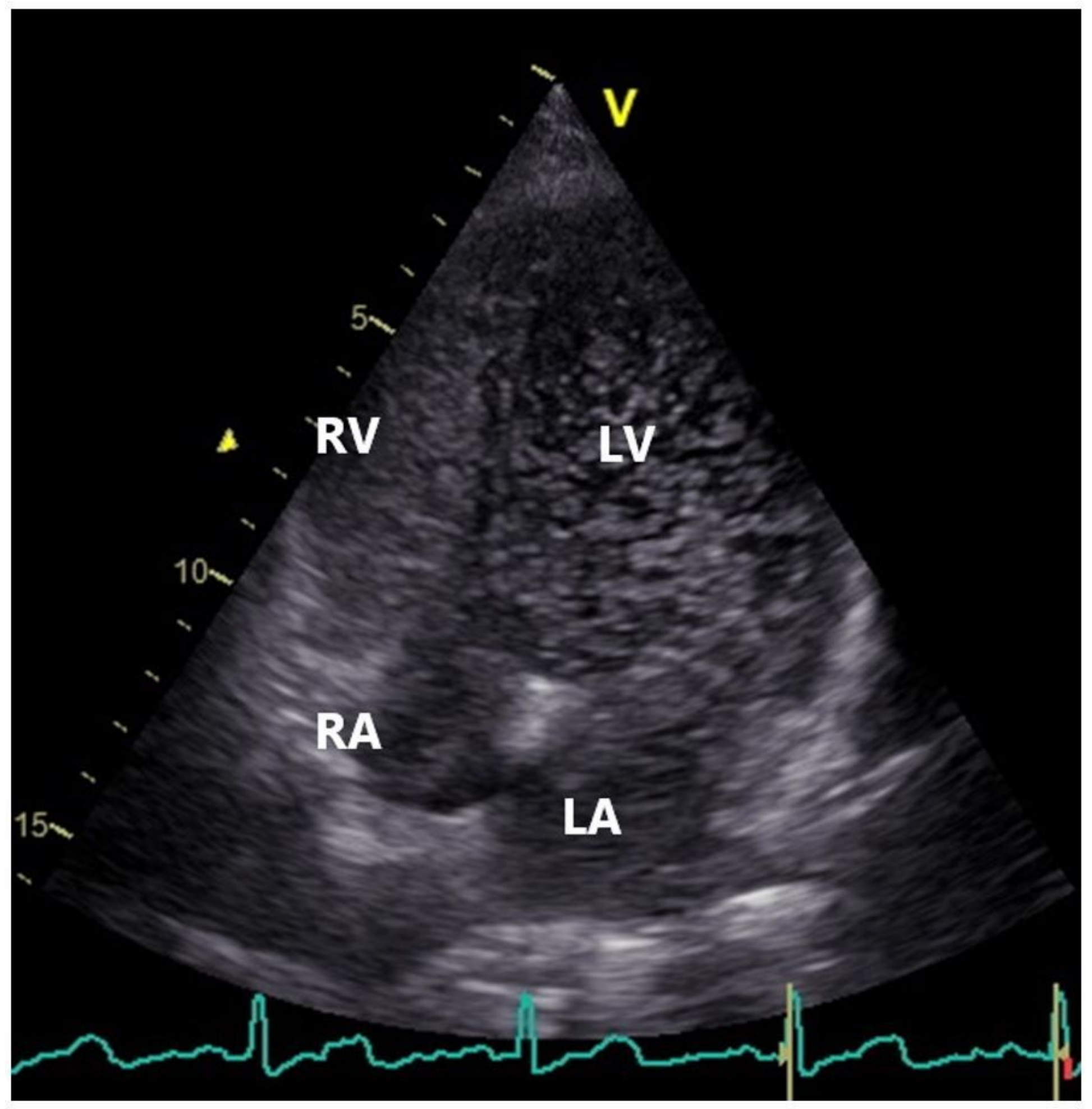

\section{Figure 7}

Contrast echocardiography showed dense microbubbles the left heart from the third cardiac cycle following the imaging of microbubbles of the right heart, suggesting pulmonary arteriovenous fistula. 

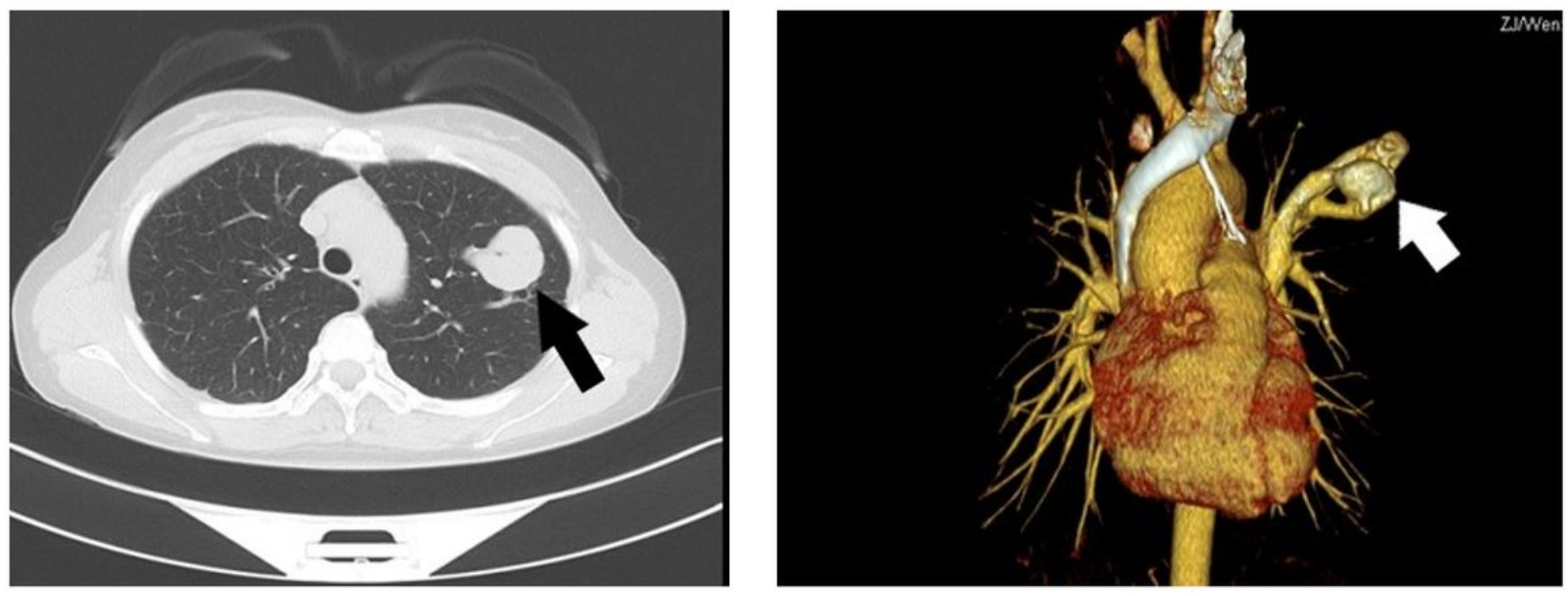

\section{Figure 9}

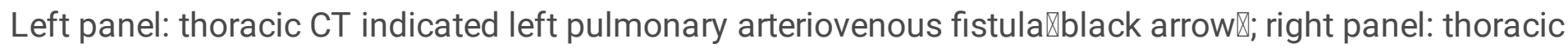
CT angiography indicated left pulmonary arteriovenous fistula (white arrow). 